University of Warwick institutional repository

This paper is made available online in accordance with

publisher policies. Please scroll down to view the document

itself. Please refer to the repository record for this item and our

policy information available from the repository home page for further information.

To see the final version of this paper please visit the publisher's website. Access to the published version may require a subscription.

Author(s): A. Grinenko, D. O. Gericke, S. H. Glenzer, and J. Vorberger Article Title: Probing the Hydrogen Melting Line at High Pressures by Dynamic Compression Year of publication: 2008 Link to published version:

http://dx.doi.org/ 10.1103/PhysRevLett.101.194801

Publisher statement: None 
A. Grinenko et al., Phys. Rev. Lett. 101, 194801 (2008)

\title{
Probing the hydrogen melting line at high pressures by dynamic compression
}

\author{
A. Grinenko, ${ }^{1}$ D.O. Gericke,${ }^{1}$ S.H. Glenzer, ${ }^{2}$ and J. Vorberger $^{1}$ \\ ${ }^{1}$ Centre for Fusion, Space and Astrophysics, \\ Department of Physics, University of Warwick, \\ Coventry CV4 7AL, United Kingdom \\ ${ }^{2}$ L-399, Lawrence Livermore National Laboratory, \\ P.O. Box 808, Livermore, CA 94551, USA
}

(Dated: August 27, 2009)

\begin{abstract}
We investigate the capabilities of dynamic compression by intense heavy ion beams to yield information about the high pressure phases of hydrogen. Employing $a b$ initio simulations and experimental data, a new wide range equation of state for hydrogen is constructed. The results show that the melting line up to its maximum as well as the transition from molecular fluids to fully ionized plasmas can be tested with the beam parameters soon to be available. We demonstrate that x-ray scattering can distinguish between phases and dissociation states.

PACS numbers: 52.27.Gr, 51.30.+i, 41.75.Ak
\end{abstract}


Experimental and theoretical investigations concerning the thermodynamic and structural properties of hydrogen continue to break new ground. Despite its seemingly simple composition, hydrogen is known to have a variety of complex phases [1]. Moreover, both experimental [2-9] and theoretical [10-16] methods have so far failed to give conclusive answers concerning the existence of a plasma phase transition, the nature of the molecular to atomic transition in the high density fluid, the location of the metallization transition in the solid, the melting line for pressures higher than $250 \mathrm{GPa}$ [17] and the question of a possible liquid at $T=0 \mathrm{~K}$ connected to it [18].

These basic questions need answers urgently as new discoveries in astrophysics and developments for inertial confinement fusion demand more accurate equation of state (EOS) data. Biased by detection methods, most extrasolar planets are giant gas planets [19, 20]. Even Jupiter and Saturn still hold secrets about their inner structure [21] that can only be revealed by combining very precise EOS data and planet modelling [22]. Similar demands on the EOS are made by simulations of inertial fusion capsules [23, 24]. Since the targets are heated and compressed from a cryogenic state, the intermediate states to the burning fusion plasma are highly correlated solids or fluids and the compression path crosses a number of known and proposed phase transitions.

Although several techniques to create high pressure samples exist, e.g., static compression in diamond anvil cells [1] and shocks driven by high-power lasers [6] or high explosives [9], the accessible parameter space is rather limited. Dynamic compression driven by intense heavy ion beams is an alternative considerably extending this space. Here, we focus on a proposed design that deposits the beam energy into a hollow cylinder (absorber) which in turn compresses the hydrogen embedded in the center $[25,26]$. We show that, by carefully tuning the beam parameters, such experiments are able to reach molecular solid and fluid phases, metallic fluids, and the region around the maximum of the melting line including parts where the melting line of hydrogen is predicted to have a negative slope [17]. Thus, our understanding of two basic phase transitions, high pressure melting and pressure ionization, can be tested. The beam parameters needed should be available with the SIS18 upgrade and are well within reach of the Facility for Antiproton and Ion Research (FAIR) being built at the Gesellschaft für Schwerionenforschung (GSI) in Darmstadt, Germany.

We perform hydrodynamic simulations to investigate the hydrogen states accessible by ion beam driven compression using our Lagrangian-Eulerian remap code. The numerical 
scheme is explicit, in second order accurate, and uses the standard arbitrary viscosity term for shock smoothing [27]. The remap step allows to control the grid at every time step using the second order Van Leer advection algorithm [28]. It is purely geometric; the physics is fully included in the Lagrangian step. The code allows for remapping to any arbitrary constant or moving grid that satisfies the stability conditions. In our simulations, the absorber-hydrogen boundary was constantly tracked and a new smoothed grid was calculated every time step such that each grid cell contains one material only.

The beam parameters and target dimensions used here are similar to those in Ref. [26]. The target consists of the absorber in the form of a hollow cylinder with an inner radius of $0.4 \mathrm{~mm}$, an outer radius of $3.0 \mathrm{~mm}$ and a length of $1 \mathrm{~cm}$. This shell is filled with cryogenic hydrogen initially at $T=14 \mathrm{~K}$ and $\rho=0.088 \mathrm{~g} / \mathrm{cm}^{3}$ corresponding to the melting point at ambient pressure. An annular beam of uranium ions with an energy of $2.7 \mathrm{GeV}$ per nucleon, an inner radius of $0.6 \mathrm{~mm}$ and an outer radius of $1.6 \mathrm{~mm}$ heats the absorber only which subsequently expands and compresses the hydrogen inside. The number of ions has been varied over two orders of magnitude around $10^{11}$ particles per bunch with a pulse duration of $20 \mathrm{~ns}$.

The energy deposition of the beam ions in the absorber was modelled using the SRIM (Stopping \& Range of Ions in Matter) tables [29]. We consider very energetic ions with stopping ranges much longer than the target length which results in a rather uniform heating of the absorber. Accordingly, the hydrodynamic motion will obey a cylindrical symmetry which justifies our use of a 1D code.

The main input quantity for our hydro-simulations is the hydrogen EOS. Since the compression starts from frozen samples, the EOS must cover solids, fluids and high density plasmas and the transitions between these phases. Except the SESAME tables [30] such an EOS did not exist. We therefore constructed a new wide range EOS using largely experimental data or first principle simulations for the important regions. Uncritical parts are calculated by classical Monte Carlo simulations using potentials fitted to experiments. In agreement with first principle simulations $[15,31]$, the obtained EOS predicts no plasma phase transition or a phase transition connected to the dissociation of molecules in the fluid.

The density-temperature region covered by our EOS is shown in Fig. 1. It spans eight orders of magnitude in the pressure and is free of adjustable parameters. In the different regions, the EOS was determined as follows: 


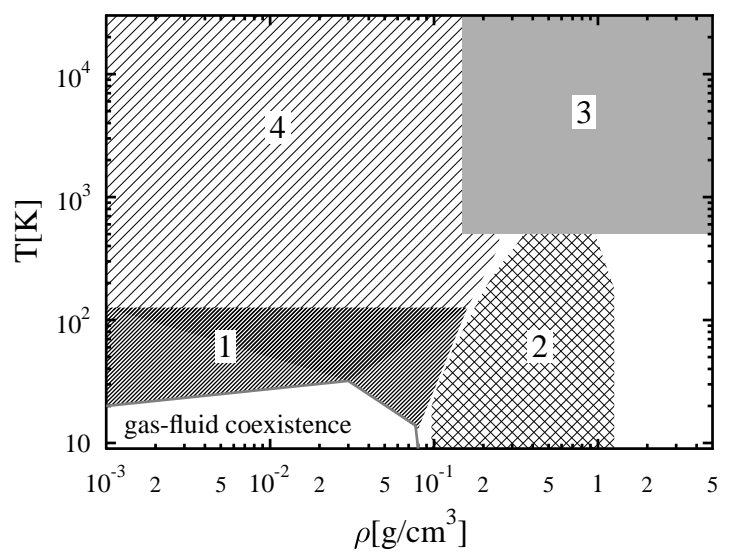

FIG. 1: Density-temperature grid for the hydrogen EOS: NIST and MC data were used in area 1. The solid hydrogen of region 2 was modeled as described in the text. DFT-MD simulations were used in region 3. The Saumon \& Chabrier EOS covers region 4. The gas-fluid coexistence region at low temperatures is indicated. There is general uncertainty about the EOS in the white area at the bottom-right.

For low temperatures and densities (region 1), we used experimentally well established data from NIST [32]. These points are complemented by Monte Carlo simulations [33] using the Ross-Ree-Young intermolecular potential [34]. Both methods agree very well. The NIST data include the gas-solid and gas-liquid transitions and some data above the critical point.

For the molecular solid phase (region 2 - disordered phase I), we employed a zero Kelvin isotherm which was determined experimentally up to $40 \mathrm{GPa}[35,36]$. These data were reproduced and extended to higher pressures by density functional ground state calculations (DFT) $[37,38]$. Temperature contributions (phonons) were then added by means of a Debye model with an experimentally obtained Debye temperature [36].

The high density fluid (region 3) spans molecular and metallic hydrogen. Here, the EOS was described by density functional molecular dynamics (DFT-MD) [15] which allows for a full quantum treatment of the electrons and strong correlations in the ion component.

The EOS of Saumon and Chabrier (S\&C) [39, 40] provides data for low density and high temperature systems (region 4). However, DFT-MD calculations were preferred in case the two approaches overlap.

In addition, we need data for lead and aluminum to describe the absorber. Compared to hydrogen, these materials undergo much less dramatic changes. We therefore rely on 


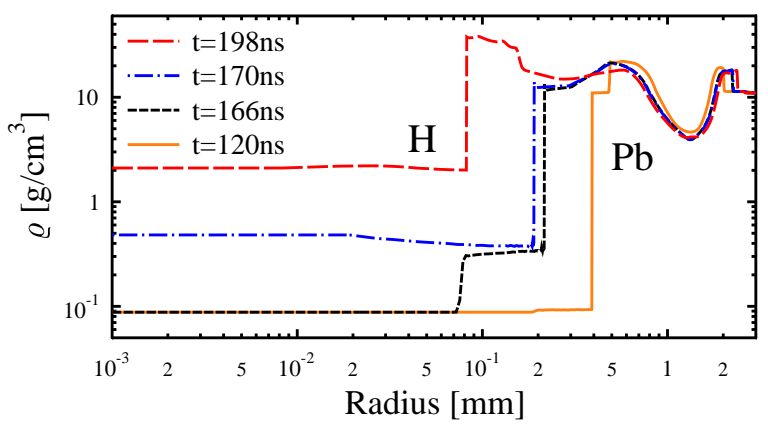

FIG. 2: (color online) Density evolution in the target at different stages of the compression for lead as the absorber material and a bunch of $10^{12}$ uranium ions.

tabulated EOS data from SESAME [30].

With these EOS data, we now perform the hydrodynamic simulations for ion beam driven compression. Fig. 2 shows a few snapshots of the density evolution in the target where time starts with the energy deposition. Since the annular ion beam is smaller than the absorber, its deposited energy results in inward and outward propagating shock waves (see first snapshot at $t=120 \mathrm{~ns})$. Naturally, the converging shock wave (SW) propagates faster and is stronger.

When the converging SW hits the material boundary at $t=166 \mathrm{~ns}$, a secondary SW is launched into the hydrogen. This SW causes the heating of the hydrogen due to the entropy jump at the SW front. After hitting the central axis, the SW is reflected and propagates back to the material boundary. At the same time, the pusher continues to expand and compresses the hydrogen core. During this slow compression, the reverberating SW inside the hydrogen increases its temperature until pressure balance is reached (at $t \approx 200 \mathrm{~ns}$ in Fig. 2).

Let us further investigate which hydrogen states can be tested by ion beam driven compression. The trajectories of fluid elements are marked by the shaded regions in Fig. 3 where the predictions of two EOS models, the SESAME tables and our newly constructed EOS, are compared. The regions are bounded by states at the absorber-hydrogen boundary from below and states located on the axis from above. The trajectories are tracked from the beginning of the compression up to the onset of the expansion of the core. The compression first appears to be Hugoniot-like, later a change in slope can be observed and the further compression is more isentropic. Clearly, the SESAME data predict considerably 


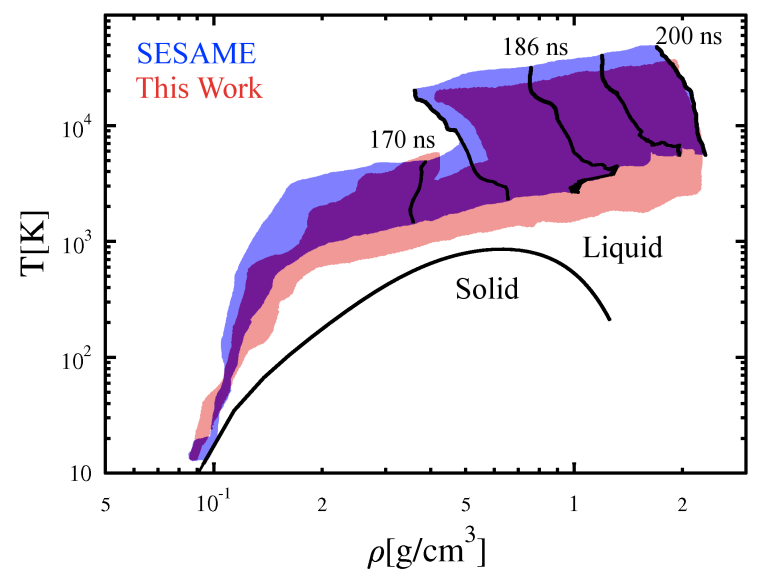

FIG. 3: (color online) Trajectories of hydrogen fluid elements for two EOS models. The lead absorber is heated by bunch of $10^{12}$ ions. The hydrogen melting line and states that occur simultaneously are marked by black lines.

hotter hydrogen than the new model related to differences in the high temperature/high density region. These differences become much smaller for lower beam intensities (almost negligible for $10^{11}$ beam ions).

For the high beam intensities used in Fig. 3, the first SW already melts the cryogenic hydrogen which becomes a molecular fluid. This region is of high astrophysical relevance since Jupiter's isentrope [22] runs through the parameters tested. On the other hand, the described mix of shock and isentropic compression makes it possible to closely follow the melting line up to its maximum and beyond with carefully chosen beam intensities (see Fig. 4). Thus, such easier to achieve beam parameters may be preferred if one is interested in probing the vicinity of the melting line. Since different EOS models predict melting at quite different positions, this gives an excellent opportunity to distinguish between them. It is particularly interesting that the theoretically predicted change in the slope of the melting line [17] is here in reach of an experimental test not achievable with diamond cells $[4,5]$. It is also complementary information to data obtained by explosive compression of hydrogen $[9]$.

We further investigated different materials as absorber. Fig. 4 shows data for lead and aluminum. Clearly, the heavier lead yields higher compression, needed to test the metallic phase, and more homogeneous temperature distributions. The aluminum EOS is, however, much better understood which gives an advantage for design studies. Again, these differences 


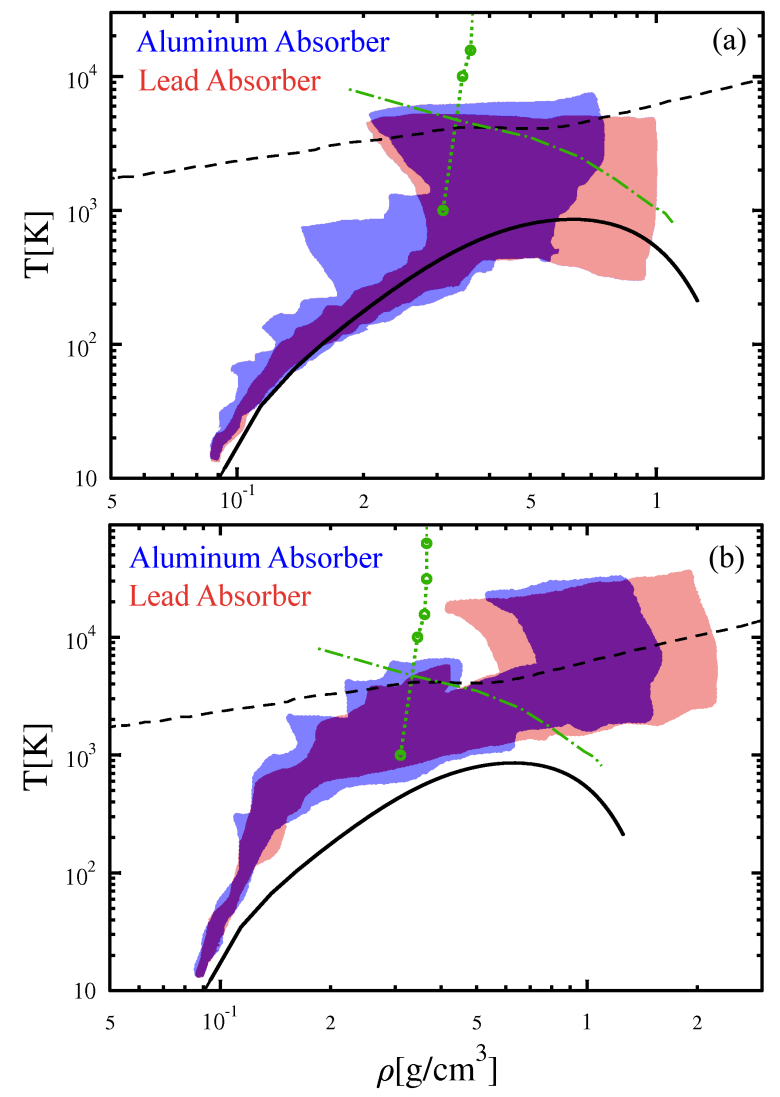

FIG. 4: (color online) Parameters reached in the hydrogen for two absorbers and two different beam intensities: (a) $10^{11}$ uranium ions and (b) $10^{12}$ ions. Shown are the melting line (solid), the isentrope of Jupiter (dashed) [22], states reached by compression with high explosives (dotted) [9], and the transition from molecular to metallic hydrogen (dash-dotted).

become more pronounced for higher beam intensities.

The structural differences between the phases offer an excellent opportunity to be tested by x-ray scattering [41-43]. Structure factors obtained by DFT-MD simulations are shown in Fig. 5 for a density where the slope of the melting line is negative. Most prominent is the peak at $4.5 \AA^{-1}$ whose height indicates order and the presence of molecules. This makes x-ray scattering very sensitive to melting and dissociation (Mott transition) as the elastic scattering peak is proportional to $S_{i i}(k)$. Moreover, the Compton and plasmon shifts give the density while the width of the Compton line yields the temperature.

The attenuation length of $3 \mathrm{keV}$ x-rays in lead is only $\sim 10 \mu \mathrm{m}$ which prohibits probing through the lead, but forward scattering through the ends of the cylinder can be performed. In cases, where less compression and temperature control is sufficient, the use of aluminum 


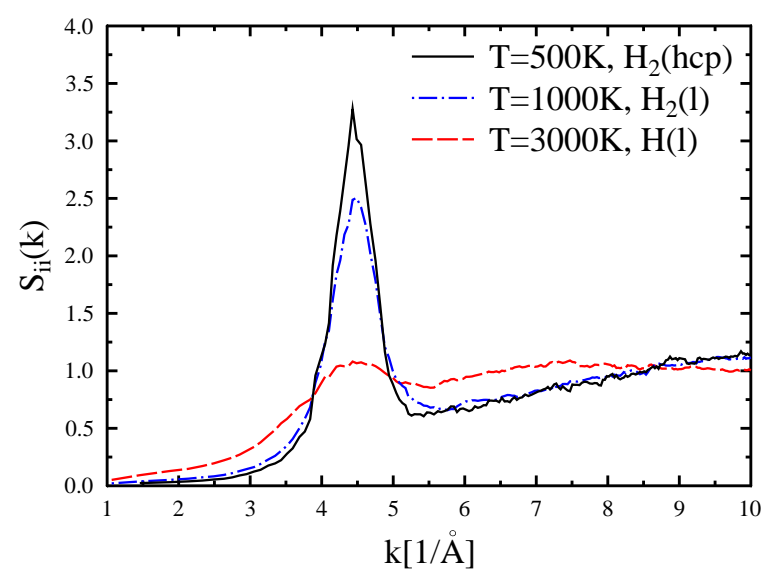

FIG. 5: (color online) Ionic structure factors for hydrogen at $P=138 \mathrm{GPa}\left(\rho=0.8 \mathrm{~g} / \mathrm{cm}^{3}\right)$ corresponding to the high pressure molecular solid $(T=500 \mathrm{~K})$, the molecular liquid $(T=1000 \mathrm{~K})$, and metallic liquid $(T=3000 \mathrm{~K})$.

can be advantageous as it is transparent for x-rays.

We performed a detailed numerical analysis for a high energy density experiment which employed a new hydrogen EOS, largely build from experimental data and first principle simulations. We showed that high pressure melting and the molecular to metallic transition are well in experimental reach with facilities soon available. In particular, the maximum and negative slope of the melting line can be tested. Moreover, our analysis shows that x-ray scattering is an excellent tool to distinguish the different phases by their structures. Thus, valuable information on the structural and thermodynamic properties of hydrogen can be obtained by dynamic compression with heavy ion beams.

Support from the EPSRC of the United Kingdom is gratefully acknowledged. The work of SHG was performed under the auspices of the U.S. Department of Energy by Lawrence Livermore National Laboratory under Contract DE-AC52-07NA27344.

[1] H. Mao and R.J. Hemley, Rev. Mod. Phys. 66, 671 (1994).

[2] R.J. Hemley et al., Phys. Rev. Lett. 76, 1667 (1996).

[3] P. Loubeyre et al., Nature 416, 613 (2002).

[4] E. Gregoryanz et al., Phys. Rev. Lett. 90, 175701 (2003).

[5] S. Deemyad and I.F. Silvera, Phys. Rev. Lett. 100, 155701 (2008). 
[6] S.T. Weir et al., Phys. Rev. Lett. 76, 1860 (1996).

[7] G.W. Collins et al., Phys. Rev. Lett. 87, 165504 (2001).

[8] M.D. Knudson et al., Phys. Rev. Lett. 90, 035505 (2003).

[9] V.E. Fortov et al., Phys. Rev. Lett. 99, 185001 (2007).

[10] G.E. Norman and A.N. Starostin, High Temp. 6, 394 (1968).

[11] W. Ebeling and W. Richert, Phys. Lett. A 108, 80 (1985).

[12] D. Saumon and G. Chabrier, Phys. Rev. Lett. 62, 2397 (1989).

[13] D. Beule, et al., Phys. Rev. B 59, 14177 (1999).

[14] S. Scandolo, Proc. Nat. Acad. Sci. USA 100, 3051 (2003).

[15] J. Vorberger et al., Phys. Rev. B 75, 024206 (2007).

[16] B. Jakob et al., Phys. Rev. E 76, 036406 (2007).

[17] S.A. Bonev et al., Nature 431, 669 (2004).

[18] E. Babaev et al., Nature 431, 666 (2004).

[19] M. Mayor and D. Queloz, Nature 378, 355 (1995).

[20] A. Burrows, Nature 433, 261 (2005).

[21] T. Guillot el al., in Jupiter, edited by F. Bagenal (Univ. of Arizona Press, Tucson, 2003), chap. 3, pp. 35-57.

[22] B. Militzer et al., accepted for Astro. Phys. J. Lett.

[23] J.D. Lindl et al., Phys. Plasmas 11, 339 (2004).

[24] S. Atzeni and J. Meyer-ter-Vehn, The Physics of Inertial Fusion (Clarendon Press, Oxford, 2004).

[25] N.A. Tahir et al., Phys. Rev. E 63, 016402 (2000).

[26] N.A. Tahir et al., Phys. Rev. B 67, 184101 (2003).

[27] A.A. Samarskii and Y.P. Popov, Difference Methods for the Solution of Problems of Gas Dynamics (Nauka, Moscow, 1980) (in Russian).

[28] B. van Leer, J. Comput. Phys. 135, 299 (1997).

[29] J. Ziegler, SRIM 2006.2., http://www.srim.org.

[30] G.I. Kerley, Los Alamos Scientific Laboratory Report No. LA-4776, UC-34.

[31] J. Vorberger et al., Contrib. Plasma Phys. 47, 375 (2007).

[32] E.W. Lemmon et al., in NIST Standard Reference Database 69 (NIST, Gaithersburg, 2005). 
[33] B. Militzer, Coulomb Monte Carlo (CMC)

[34] M. Ross et al., J. Chem. Phys. 79, 1487 (1983).

[35] V. Diatschenko et al., Phys. Rev. B 32, 381 (1985).

[36] R.J. Hemley et al., Phys. Rev. B 42, 6458 (1990).

[37] M. Fuchs and M. Scheffler, Comp. Phys. Com. 119, 67 (1999).

[38] Copyright ABINIT group (M. Mikami et al.) 2003-2005.

[39] D. Saumon and G. Chabrier, Phys. Rev. A 46, 2084 (1992).

[40] D. Saumon, G. Chabrier, and H.M. Van Horn, Astrophys. J. Suppl. 99, 713 (1995).

[41] S.H. Glenzer et al., Phys. Rev. Lett. 90, 175002 (2003).

[42] A.L. Kritcher et al., Science 322, 69 (2008).

[43] E. García Saiz et al., Nature Physics 4, 940 (2008). 